Check for updates

Cite this: Chem. Sci., 2019, 10, 1831

๑ All publication charges for this article have been paid for by the Royal Society of Chemistry

Received 19th September 2018 Accepted 30th November 2018

DOI: $10.1039 / \mathrm{c} 8 \mathrm{sc} 04169 \mathrm{e}$

rsc.li/chemical-science

\section{Specific recognition of proteins and peptides via controllable oriented surface imprinting of boronate affinity-anchored epitopes $\uparrow$}

\author{
Rongrong Xing, (D) Yanyan Ma, Yijia Wang, Yanrong Wen and Zhen Liu (DD *
}

\begin{abstract}
Molecularly imprinted polymers (MIPs) are chemically synthesized materials mimicking the recognition of antibodies towards antigens. Epitope imprinting has been an effective strategy, making imprinting of proteins flexible to a great extent. However, so far there is apparently a lack of facile and versatile epitope imprinting approaches. Herein, we present a new method called controllable oriented surface imprinting of boronate affinity-anchored epitopes. In this method, a C-terminus nonapeptide epitope was glycated and anchored as a template onto a boronic acid-functionalized substrate, followed by controllable oriented surface imprinting via the polycondensation of multiple silylating reagents containing functionalities capable of interacting with the epitope. The developed imprinting approach allowed for precise control of the thickness of the imprinting layer through adjusting the imprinting time, generating excellent binding properties. This method was verified to be versatile and efficient. Thus, it could greatly facilitate the preparation of MIPs for specific recognition of proteins and peptides.
\end{abstract}

\section{Introduction}

Due to the presence of imprinted cavities complementary to the shape and functionalities of template molecules, molecularly imprinted polymers (MIPs) ${ }^{1-7}$ can provide affinity and specificity toward the template molecules and thereby have found promising applications in many areas such as sensing, ${ }^{\mathbf{8} 9}$ separation $^{10,11}$ and catalysis. ${ }^{12,13}$ As compared with antibodies and enzymes, MIPs are easier to prepare, more cost-efficient and more stable. So far, a large number of imprinting approaches have been reported. However, the imprinting of biomacromolecules, especially proteins, still remains challenging. Under harsh polymerization conditions for imprinting, a severe conformational change of proteins often occurs. In addition, the large size of proteins makes them difficult to be removed from highly cross-linked polymer networks. Epitope imprinting for peptide recognition was pioneered by Rachkov and Minoura. ${ }^{\mathbf{1 4 , 1 5}}$ In epitope imprinting, short characteristic peptides, rather than entire proteins, are used as templates. A milestone study in epitope imprinting from shea and coworkers ${ }^{\mathbf{1 6}}$ extended this strategy to protein recognition, in which Cterminal nonapeptides were selected as epitopes. Since then, epitope imprinting has been widely adopted to overcome the above issues in protein imprinting. ${ }^{17-22}$ However, conventional

State Key Laboratory of Analytical Chemistry for Life Science, School of Chemistry and Chemical Engineering, Nanjing University, 163 Xianlin Avenue, Nanjing 210023, China.E-mail: zhenliu@nju.edu.cn; Fax:+86-25-8968-5639

$\dagger$ Electronic supplementary information (ESI) available. See DOI: $10.1039 / \mathrm{c} 8 \mathrm{sc} 04169 \mathrm{e}$ epitope imprinting still suffers from some drawbacks. In some methods, prior to the imprinting the epitopes are immobilized on substrates via a functionality on the terminal amino acids such as $-\mathrm{SH}^{17}$ and $-\mathrm{NH}_{2} \cdot{ }^{6,18}$ However, the co-existence of the same functionality on other amino acids may lead to an unfavourable configuration of the epitope on the substrate. Also, there is a lack of generally applicable substrates for facile and efficient epitope immobilization. In some approaches, epitopes are not immobilized ${ }^{\mathbf{1 9 - 2 2}}$ and the imprinting direction is random, which results in a low imprinting efficiency since a large portion of the template is buried in the polymer. More importantly, many methods are only applicable to limited cases, and tedious conditional selection is usually required for imprinting different epitopes.

Boronic acids, which can reversibly bind with cis-diols, ${ }^{23,24}$ are important functional monomers for the imprinting of cisdiol-containing compounds such as saccharides and glycoproteins. ${ }^{25-29}$ Recently, we developed a versatile, facile and efficient approach called boronate affinity controllable oriented surface imprinting ${ }^{30-33}$ for the imprinting of glycoproteins, glycans and monosaccharides. The prepared MIPs exhibit high specificity, strong affinity and excellent imprinting efficiency. When applying to different targets, the change in the imprinting procedure is only to adjust the imprinting time according to the molecular size of the template. Clearly, an epitope imprinting approach that features the above merits, including versatility, facility, efficiency and excellent binding properties, will be of significant importance.

Herein, we present a new approach, called controllable oriented surface imprinting of boronate affinity-anchored 


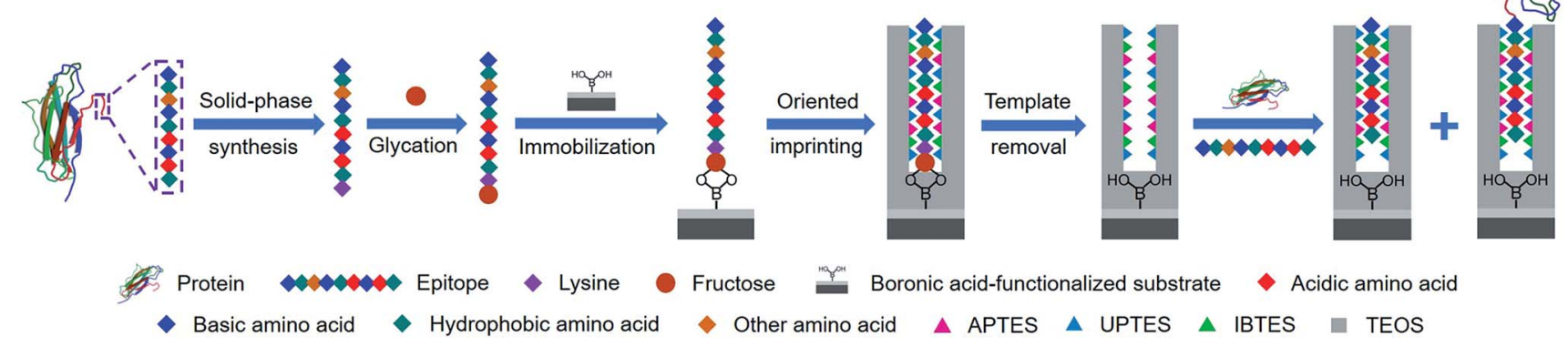

Fig. 1 Schematic of the principle and procedure of controllable oriented surface imprinting of boronate affinity-anchored epitopes.

epitopes, for the imprinting of proteins. This approach relies on the combination of glycation of epitopes, immobilization of glycated epitopes on boronic acid-functionalized substrates, and controllable oriented surface imprinting. The procedure of the imprinting is shown in Fig. 1. Based on the amino acid sequence of a specific protein, a C-terminus nonapeptide is selected as the epitope. Its C-terminus is attached with an additional lysine, followed by glycation with fructose. The glycated epitope is then immobilized onto a boronic acid-functionalized substrate by virtue of boronate affinity. After that, a silica coating is formed on the substrate surface to cover the template to an appropriate thickness, via the polycondensation of multiple silylating reagents containing functionalities capable of interacting with the template, including aminopropyltriethoxysilane (APTES), 3-ureidopropyl-triethoxysilane (UPTES), isobutyltriethoxysilane (IBTES) and tetraethyl orthosilicate (TEOS). Finally, the glycated epitope is removed by rinsing with an acidic acetonitrile solution to disrupt noncovalent and boronate affinity interactions, leaving imprinted cavities on the substrate surface. The obtained MIPs can rebind the intact target proteins and the epitope peptides. The highly attractive features of this approach, including versatility, facility, efficiency and excellent binding properties, were experimentally confirmed. Thus, it can be a promising method for the preparation of protein-specific MIPs for important applications.

\section{Results and discussion}

\section{Selection and glycation of the epitope}

$\beta_{2}$-Microglobulin (B2M) and myoglobin (Mb) were used as the target proteins while boronic acid-functionalized magnetic nanoparticles (MNPs) were employed as the substrate in this work. B2M, which consists of 99 amino acids, is one of the major histocompatibility complex class I molecules. The abnormal level of B2M can indicate different diseases such as multiple myeloma. ${ }^{34} \mathrm{Mb}$, containing 153 amino acids, is a potential marker for heart attack in patients with chest pain. ${ }^{35}$ From protein structure databases such as UniProt, C-terminal nonapeptides for human $\mathrm{B} 2 \mathrm{M}$ and $\mathrm{Mb}$ were found to be KIVKWDRDM and NYKELGFQG, respectively. The general procedure for the preparation of glycated epitope-imprinted
MNPs is shown in Fig. S1. $\dagger$ A lysine is introduced at the C-end, because its amino group can be linked with a monosaccharide through the reductive amination. Fructose was selected as the sugar moiety and 2,4-difluoro-3-formyl-phenylboronic acid (DFFPBA) was selected as the ligand, because of their strong affinity. ${ }^{36}$ The synthesis of the peptide sequences and the glycation were achieved through solid-phase synthesis. In the solid-phase synthesis of the sequence of nonapeptide plus terminal lysine, functionalities at the side chains except for the terminal lysine were all protected. Thus, the sugar moiety was only linked to the amino group of the terminal lysine. The structures of the glycated B2M epitope and glycated Mb epitope are shown in Fig. S2.†

\section{Optimization of monomer composition and imprinting time}

The boronate affinity of $\mathrm{Fe}_{3} \mathrm{O}_{4} @ \mathrm{SiO}_{2} @ D F F P B A$ MNPs was verified, as shown in Fig. S3 and S4. $\dagger$ Unlike the boronate affinity controllable oriented surface imprinting, ${ }^{30-33}$ the boronic acid moiety introduced herein functioned only as an anchor for the facile immobilization and removal of the template, rather than participating in the rebinding with the epitopes. Because of the absence of boronate affinity in the rebinding, the use of multiple silylating reagents containing functionalities capable of interacting with the epitopes was essential to gain high rebinding affinity. The combination of the silylating reagents was selected according to the common features of amino acids of peptide epitopes. As shown in Fig. S5†, APTES, which contains an amino group, can interact with class I amino acids via electrostatic attraction as well as class IV amino acids via hydrogen bonding; UPTES, which contains a carbamido group, can interact with class II amino acids and class IV amino acids mainly via hydrogen bonding; IBTES, which contains a hydrophobic chain, can interact with class III amino acids via hydrophobic interaction. Although TEOS contains no functional groups, it can function as a crosslinker to form a hydrophilic silica skeleton, which can not only crosslink other monomers but also help to reduce non-specific adsorption. The ratio of the monomers and the imprinting time was optimized in terms of the imprinting factor (IF), which was calculated according to the ratio of the amount of epitope captured by glycated epitope-imprinted MNPs over that by non-imprinted MNPs (NIPs). As shown in Table S1 $\uparrow$ and Fig. 2, the composition 


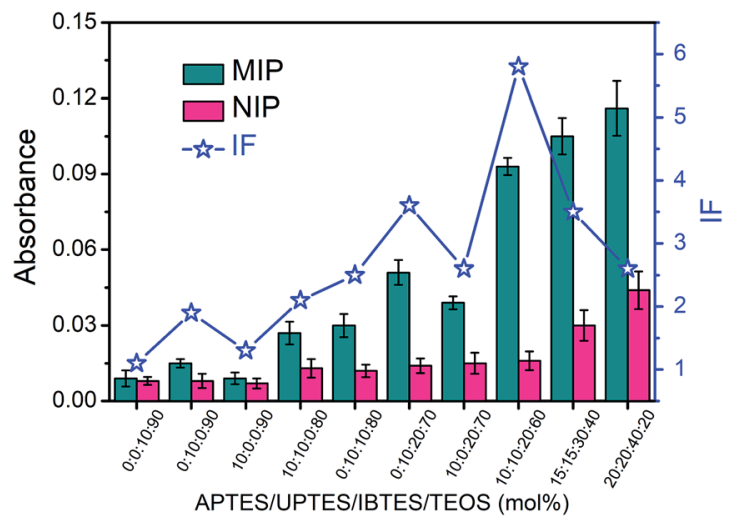

Fig. 2 The amount of the B2M epitope captured by glycated B2M epitope-imprinted MNPs and non-imprinted MNPs prepared using different proportions of monomers in an optimal imprinting time and their IF values. The error bars represent the standard deviation of three parallel experiments.

of monomers played a key role. When an unsuitable composition (such as 1-5) was used, the overall IF value was rather poor, whatever the imprinting time was. In contrast, at an appropriate composition (such as 6, 8 and 9), the overall IF value was high and the imprinting time became critical. Imprinting with composition 8 (the ratio of APTES/UPTES/IBTES/TEOS was $10: 10: 20: 60$ ) for 60 min produced the highest IF value (5.8).

\section{Characterization and performance of glycated epitope- imprinted MNPs}

As indicated by the transmission electron microscopic (TEM) images shown in Fig. S6, $\uparrow$ the glycated epitope-imprinted MNPs exhibited a diameter of $c a .150 \mathrm{~nm}$, and a thin silica layer could be seen on the surface of the MNPs. Fourier transform infrared (FT-IR) characterization confirmed that the monomers were successfully modified on the MNPs (Fig. S7†). The binding isotherm of the glycated B2M epitope-imprinted MNPs toward the B2M epitope was evaluated. The imprinted MNPs exhibited much stronger affinity toward the B2M epitope as compared with the non-imprinted MNPs (Fig. 3a). Scatchard plot analysis (Fig. 3b) gave a dissociation constant $\left(K_{\mathrm{d}}\right)$ of $(2.08 \pm 0.11) \times$ $10^{-7} \mathrm{M}$. The imprinting efficiency was measured to be $54.2 \%$ (see the ESI $\dagger$ ), which was excellent for the imprinting of
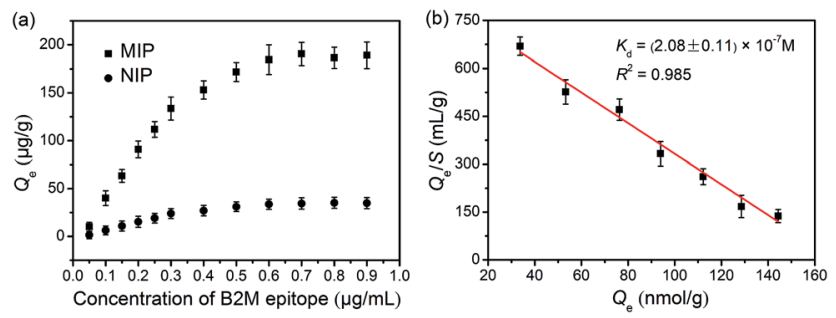

Fig. 3 (a) Binding isotherms for the binding of glycated B2M epitopeimprinted MNPs and non-imprinted MNPs to the B2M epitope. (b) Scatchard plot for the binding between glycated B2M epitopeimprinted MNPs and the B2M epitope. proteins. The extraction of the $\mathrm{B} 2 \mathrm{M}$ epitope and the $\mathrm{B} 2 \mathrm{M}$ intact protein by the glycated B2M epitope-imprinted MNPs was found to reach equilibrium within $20 \mathrm{~min}$ (Fig. S8†). Such fast equilibrium kinetics benefited from the accessible binding sites on the surface of the imprinted MNPs. Moreover, the glycated B2M epitope-imprinted MNPs could be stably stored for at least three months in a dry and sealed tube at room temperature. Even after storage for three months, the amount of B2M captured by the glycated B2M epitope-imprinted MNPs decreased only by 13.4\% (Fig. S9†).

\section{Specificity test}

The specificity of the glycated epitope-imprinted MNPs toward intact proteins was examined by using $\mathrm{B} 2 \mathrm{M}$ as the target protein, with ribonuclease A (RNase A), ribonuclease B (RNase B), horseradish peroxidase (HRP), and bovine serum albumin (BSA) as the competing proteins. As shown in Fig. 4, when the imprinting time was $60 \mathrm{~min}$, the glycated B2M epitopeimprinted MNPs exhibited the highest IF value (6.5) and the best specificity, which were consistent with the results using the B2M epitope as the target analyte. Furthermore, the glycated B2M epitope-imprinted MNPs could endure 6 consecutive uses, with cross-reactivity less than $30 \%$ (Fig. S10†). Matrix-assisted laser desorption/ionization time-of-flight mass spectrometric (MALDI-TOF MS) analyses also confirmed the specificity of the glycated epitope-imprinted MNPs (Fig. S11-S13†).

The specificity of the glycated epitope-imprinted MNPs at the peptide level was investigated. Tryptic digests of HRP and BSA were employed as the competing species. A mixture of the B2M epitope and the tryptic digests of HRP and BSA (molar ratio, $1: 1: 1)$ was extracted with the glycated B2M epitope-imprinted and non-imprinted MNPs. Without extraction, the B2M epitope, 10 peptides from BSA and 2 peptides and 8 glycopeptides from HRP were detected. However, with extraction by the glycated B2M epitope-imprinted MNPs, only the B2M epitope was detected. In contrast, nearly nothing was extracted by the non-
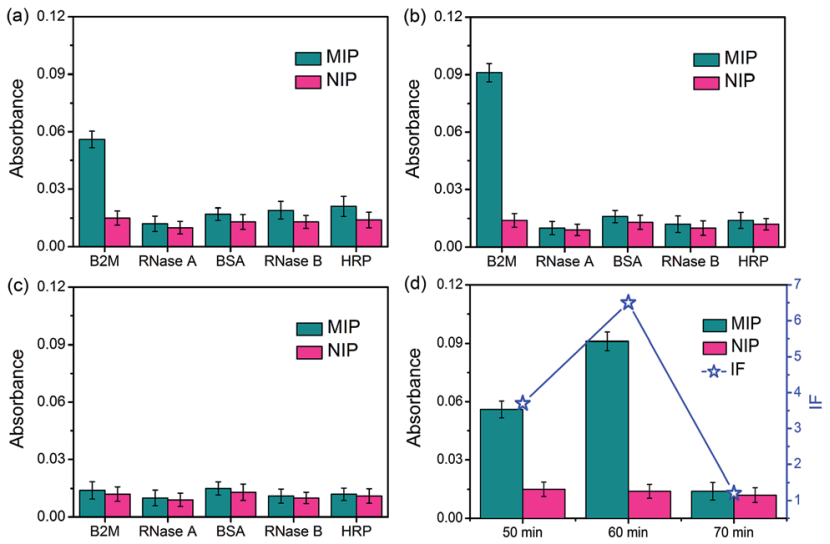

Fig. 4 The amount of different proteins captured by glycated B2M epitope-imprinted MNPs and non-imprinted MNPs prepared in the monomer ratio of APTES/UPTES/IBTES/TEOS $=10: 10: 20: 60$ with different imprinting times $(\mathrm{a}-\mathrm{c})$ and the dependence of IF on imprinting time (d). Imprinting time: (a) $50 \mathrm{~min}$; (b) $60 \mathrm{~min}$; (c) $70 \mathrm{~min}$. 
imprinted MNPs (Fig. S14 and Table S2 $\dagger$ ). Clearly, the glycated epitope-imprinted MNPs exhibited high specificity at the peptide level.

\section{Thickness controllability characterization of the imprinting layer}

The dependence of the thickness of the imprinting layer on the imprinting time was examined. As shown in Fig. 5, the thickness of the imprinting layer linearly increased with increase in the polymerization time $(y=(0.066 \pm 0.003) x+(0.161 \pm 0.183)$, $\left.R^{2}=0.988\right)$. This indicates good controllability of the polymerization system used. The performance of the glycated epitope-imprinted MNPs prepared at different imprinting times can be rationalized in terms of the template coverage, which is the ratio of the thickness of the imprinting layer over the total length of the template and the ligand. With the software ChemBio 3D, the length of the glycated B2M epitope plus the boronic acid ligand was calculated to be $c a .4 .44 \mathrm{~nm}$. Using the above linear equation, the template coverage was calculated to be 78,93 , and $108 \%$ for the imprinting times of 50, 60 and 70 min, respectively. At relatively low coverage (such as $78 \%$ ), the thickness of the imprinting layer was insufficient, resulting in relatively poor affinity and specificity. When the coverage was over $100 \%$, the template molecules were all buried within the imprinting layer, making the MIP function like a NIP and thereby showing no affinity and specificity. Theoretically, the best imprinting time should be the value at which the thickness of the imprinting layer is close to but less than $100 \%$. Clearly, the coverage at the imprinting time of $60 \mathrm{~min}$ was close to the theoretical optimum. The above rationalization suggests that the imprinting system used herein allowed for precision control of the imprinting process and thereby provided the best binding performance.

\section{Real sample application}

The potential of the glycated epitope-imprinted MNPs for realworld application was demonstrated by the extraction of B2M directly from human serum. MALDI-TOF MS analyses indicated that B2M existed in low abundance in human serum, accompanied by abundant interfering proteins, such as human serum
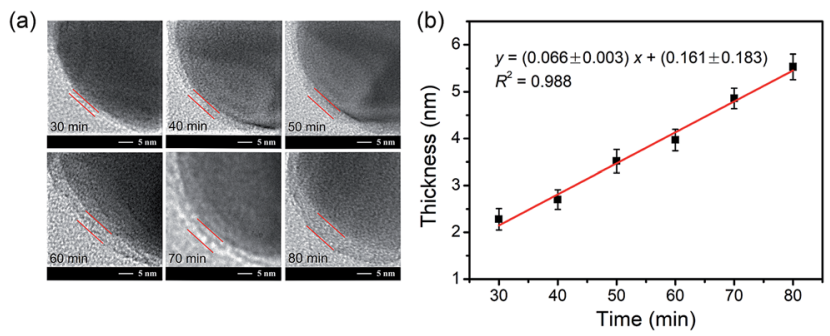

Fig. 5 (a) TEM images for the imprinting layer of AgNPs at the monomer ratio of APTES/UPTES/IBTES/TEOS $=10: 10: 20: 60$ at different polymerization times. Scale bar $=5 \mathrm{~nm}$. (b) Relationship between the thickness of the imprinting layer and polymerization time. The ratio of APTES/UPTES/IBTES/TEOS was $10: 10: 20: 60$. The error bars represent the standard deviation of five parallel experiments. albumin (HSA). As a comparison, after the serum sample was treated with the glycated B2M epitope-imprinted MNPs, only enriched B2M was detected (Fig. S15 $\dagger$ ). To further investigate the properties of the glycated epitope-imprinted MNPs, the tryptic digests of human serum samples spiked with the B2M epitope were analyzed. Even though there were plenty of interfering species, only the B2M epitope was detected by MALDITOF MS (Fig. 6). Apparently, the glycated epitope-imprinted MNPs exhibited excellent specificity toward both the intact target protein and its epitope even in a complex sample matrix.

\section{Method versatility}

The versatility of this approach was demonstrated by applying the developed method to the imprinting of $\mathrm{Mb}$. Although the ratio of the silylating monomers optimized for the B2M epitope might not be the best for the imprinting of the Mb epitope, the combination should be applicable to the imprinting of the $\mathrm{Mb}$ epitope and other epitopes, since the combination of the silylating monomers was selected according to the common features of amino acids. On the other hand, for nonapeptide epitopes glycated with the same monosaccharide through the linkage of the same additional amino acid, the molecular lengths are the same. Therefore, if the same composition of the polymerization system is used, the imprinting time optimized for one epitope can be applied to another. Thus, composition 8 (APTES/UPTES/IBTES/TEOS = $10: 10: 20: 60$ ) was used and the imprinting time was set at $60 \mathrm{~min}$. The prepared glycated $\mathrm{Mb}$ epitope-imprinted MNPs exhibited excellent specificity at both the intact protein level and the peptide level (Fig. S16-S18,
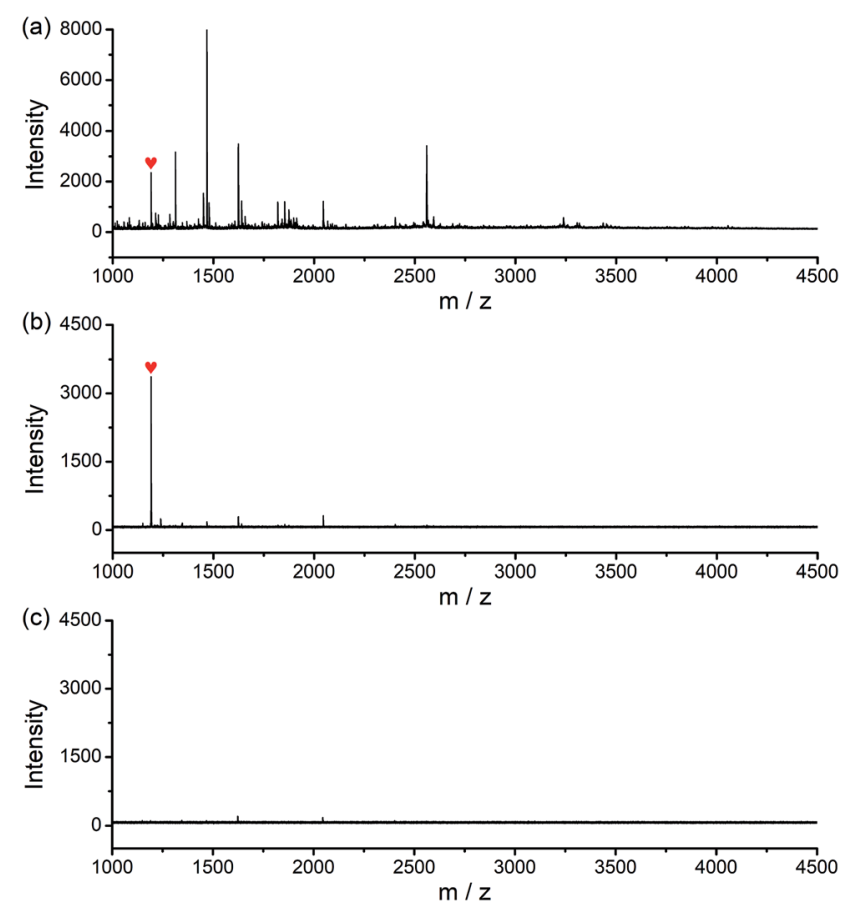

Fig. 6 MALDI-TOF MS spectra for the analysis of the B2M epitope spiked tryptic digest of human serum. (a) Without, (b) with extraction by glycated B2M epitope-imprinted MNPs and (c) with extraction by the corresponding non-imprinted MNPs ( $\mathbf{v}$ : B2M epitope). 
Table S3 $\dagger$ ). The IF value was 5.6, and the imprinting efficiency was $52.6 \%$ (see the ESI $\dagger$ ).

\section{Conclusions}

In summary, we developed a versatile, facile and efficient approach for the imprinting of proteins. Through the introduction of a sugar moiety into the epitope, the immobilization and release of the templates became straightforward through manipulating the molecular interactions involved. With the immobilized templates and the silane-based imprinting system, the imprinting process was performed in a manner of precisely controllable oriented surface imprinting, providing strong affinity, high specificity and excellent efficiency. Thus, the proposed imprinting approach provided new access to the facile preparation of MIPs that allow for the specific recognition of proteins and peptides.

\section{Conflicts of interest}

There are no conflicts to declare.

\section{Acknowledgements}

We acknowledge financial support from the National Science Fund for Distinguished Young Scholars from the National Natural Science Foundation of China (21425520 and 21834003), the "333" Talents Project from the Jiangsu Provincial Government, China (BRA2016351), the Open Grant from the State Key Laboratory of Analytical Chemistry for Life Science (5431ZZXM1706), and the program A for Outstanding PhD candidate of Nanjing University (201801A006).

\section{Notes and references}

1 G. Wulff and A. Sarhan, Angew. Chem., Int. Ed. Engl., 1972, 11, 341-344.

2 G. Vlatakis, L. I. Andersson, R. Müller and K. Mosbach, Nature, 1993, 361, 645-647.

3 L. Ye and K. Mosbach, J. Am. Chem. Soc., 2001, 123, 29012902.

4 J. Svenson, N. Zheng and I. A. Nicholls, J. Am. Chem. Soc., 2004, 126, 8554-8560.

5 A. Cutivet, C. Schembri, J. Kovensky and K. Haupt, J. Am. Chem. Soc., 2009, 131, 14699-14702.

6 Y. Hoshino, H. Koide, T. Urakami, H. Kanazawa, T. Kodama, N. Oku and K. J. Shea, J. Am. Chem. Soc., 2010, 132, 66446645.

7 J. K. Awino and Y. Zhao, J. Am. Chem. Soc., 2013, 135, 1255212555.

8 A. Kugimiya and T. Takeuchi, Biosens. Bioelectron., 2001, 16, 1059-1062.

9 Y. Fuchs, O. Soppera, A. G. Mayes and K. Haupt, Adv. Mater., 2013, 25, 566-570.

10 B. R. Hart, D. J. Rush and K. J. Shea, J. Am. Chem. Soc., 2000, 122, 460-465.
11 A. Nematollahzadeh, W. Sun, C. S. A. Aureliano, D. Ltkemeyer, J. Stute, M. J. Abdekhodaie, A. Shojaei and B. Sellergren, Angew. Chem., Int. Ed., 2011, 50, 495-498.

12 G. Wulff, Chem. Rev., 2002, 102, 1-28.

13 Z. J. Zhang and J. W. Liu, Mater. Horiz., 2018, 5, 738-744.

14 A. Rachkov and N. Minoura, J. Chromatogr. A, 2000, 889, 111118.

15 A. Rachkov and N. Minoura, Biochim. Biophys.Acta, 2001, 1544, 255-266.

16 H. Nishino, C. S. Huang and K. J. Shea, Angew. Chem., Int. Ed., 2006, 45, 2392-2396.

17 D. Dechtrirat, K. J. Jetzschmann, W. F. M. Stöcklein, F. W. Scheller and N. Gajovic-Eichelmann, Adv. Funct. Mater., 2012, 22, 5231-5237.

18 A. N. Tang, L. P. Duan, M. J. Liu and X. C. Dong, J. Mater. Chem. B, 2016, 4, 7464-7471.

19 C. H. Lu, Y. Zhang, S. F. Tang, Z. B. Fang, H. H. Yang, X. Chen and G. N. Chen, Biosens. Bioelectron., 2012, 31, 439-444.

20 A. M. Bossi, P. S. Sharma, L. Montana, G. Zoccatelli, O. Laub and R. Levi, Anal. Chem., 2012, 84, 4036-4041.

21 P. Palladino, M. Minunni and S. Scarano, Biosens. Bioelectron., 2018, 106, 93-98.

22 Y. Q. Yang, X. W. He, Y. Z. Wang, W. Y. Li and Y. K. Zhang, Biosens.Bioelectron., 2014, 54, 266-272.

23 S. D. Bull, M. G. Davidson, J. M. H. Van den Elsen, J. S. Fossey, A. T. A. Jenkins, Y. B. Jiang, Y. Kubo, F. Marken, K. Sakurai, J. Z. Zhao and T. D. James, Acc. Chem. Res., 2013, 46, 312-326.

24 D. J. Li, Y. Chen and Z. Liu, Chem. Soc. Rev., 2015, 44, 80978123.

25 L. Li, Y. Lu, Z. J. Bie, H. Y. Chen and Z. Liu, Angew. Chem., Int. Ed., 2013, 52, 7451-7454.

26 Y. X. Li, M. Hong, M. Miao, Q. Bin, Z. Y. Lin, Z. W. Cai and G. N. Chen, J. Mater. Chem. B, 2013, 1, 1044-1051.

27 W. Zhang, W. Liu, P. Li, H. B. Xiao, H. Wang and B. Tang, Angew. Chem., Int. Ed., 2014, 53, 12489-12493.

28 A. Stephenson-Brown, A. L. Acton, J. A. Preece, J. S. Fossey and P. M. Mendes, Chem. Sci., 2015, 6, 5114-5119.

29 J. K. Awino, R. W. Gunasekara and Y. Zhao, J. Am. Chem. Soc., 2016, 138, 9759-9762.

30 R. R. Xing, S. S. Wang, Z. J. Bie, H. He and Z. Liu, Nat. Protoc., 2017, 12, 964-987.

31 S. S. Wang, J. Ye, Z. J. Bie and Z. Liu, Chem. Sci., 2014, 5, 1135-1140.

32 Z. J. Bie, Y. Chen, J. Ye, S. S. Wang and Z. Liu, Angew. Chem., Int. Ed., 2015, 54, 10211-10215.

33 S. S. Wang, D. Y. Yin, W. J. Wang, X. J. Shen, J. J. Zhu, H. Y. Chen and Z. Liu, Sci. Rep., 2016, 6, 22757.

34 S. Gatto, G. Ball, F. Onida, H. M. Kantarjian, E. H. Estey and M. Beran, Blood, 2013, 102, 1622-1625.

35 M. Weber, M. Rau, K. Madlener, A. Elsaesser, D. Bankovic, V. Mitrovic and C. Hamm, Clin. Biochem., 2005, 38, 10271030.

36 C. C. Lü, H. Y. Li, H. Y. Wang and Z. Liu, Anal. Chem., 2013, 85, 2361-2369. 